Check for updates

Cite this: RSC Adv., 2017, 7, 42541

\title{
Hydrogels with high mechanical strength cross- linked by a rosin-based crosslinking agent
}

\begin{abstract}
Haibo Zhang, ${ }^{\text {ab }}$ Xin Huang, ${ }^{\text {*ac }}$ Jianxin Jiang, ${ }^{b}$ Shibin Shang (iD *ac and Zhanqian Song ${ }^{a}$
In recent years, double-network (DN) hydrogels with high mechanical strength and toughness have been developed as promising materials, such as absorbents, tissue scaffolds and wastewater treatment agents. Herein, we present a novel DN hydrogels, which were prepared by micellar copolymerization of acrylamide (AM) and a rosin-based crosslinking agent (FPA-PEG200-AC) in a micellar solution of sodium dodecyl sulfate (SDS). The hydrogels using a rosin-based crosslinking agent (RPAM hydrogels) could form both chemical crosslinks and crosslinked centers for hydrophobic association to enhance mechanical strength. The formulated hydrogels exhibited high elongation ratios at break (500-1500\%) and excellent compressive strain (>95\%). Also, with an increase in the amount of crosslinking agent, the elongation at break and swelling ratio decreased. The surface morphology of the hydrogels was observed by scanning electron microscopy (SEM), while the thermal properties were characterized by thermogravimetric analysis. The results indicate a successful synthesis of the double-network (DN) hydrogels with high mechanical strength. The RPAM DN hydrogel synthesis can open fresh avenues for the design and construction of DN systems, broadening the scope of the current research and applications of hydrogels with excellent mechanical properties.
\end{abstract}

Received 20th July 2017

Accepted 23rd August 2017

DOI: $10.1039 / c 7 r a 08024 g$

rsc.li/rsc-advances

\section{Introduction}

Hydrogels consist of a three-dimensional polymer network which contains a large amount of water while maintaining its structure. Due to a combination of solid and liquid components, hydrogels have been used in diverse applications, such as wastewater treatment, ${ }^{1}$ agriculture, ${ }^{2}$ absorbents, ${ }^{3}$ tissue scaffolds,${ }^{4}$ and drug delivery. ${ }^{5}$ Hydrogels containing a large amount of water have great potential in the replacement of bio-tissues. However, many tissues such as muscle, ligament, cartilage and skin exhibit an excellent toughness, softness and mechanical properties. ${ }^{6-8}$ The classic hydrogels deliver a poor mechanical performance because of the lack of efficient energy dissipation in their gel network; ${ }^{9,10}$ resulting in limiting their applications as biomaterials in biological studies. Therefore, preparation of hydrogels with good mechanical properties is important. To obtain hydrogels with high mechanical strength, hydrogels with different network structures, such as double network (DN) hydrogels, ${ }^{11}$ sliding-ring hydrogels, ${ }^{12}$ macromolecular microsphere composite hydrogels, ${ }^{13}$ nanocomposite (NC) hydrogels, ${ }^{14}$ and physical networks, ${ }^{15,16}$ have been fabricated.

${ }^{a}$ Institute of Chemical Industry of Forest Products, CAF, National Engineering Lab. for Biomass Chemical Utilization, Key and Open Lab. of Forest Chemical Engineering, SFA, Key Lab. of Biomass Energy and Material, Jiangsu Province, Nanjing 210042, China.E-mail: shangsb@hotmail.com

${ }^{b}$ College of Materials Science and Technology, Beijing Forestry University, Engineering Research Center of Forestry Biomass Material and Bioenergy, Ministry of Education, Beijing 100083, China

'Institute of New Technology of Forestry, CAF, Beijing 100091, China
DN hydrogels, composed of loosely and tightly linked networks, ${ }^{17}$ have demonstrated excellent mechanical properties. These two structures and their interactions have an important effect on the mechanical properties of hydrogels. The loosely linked network is formed by physical interactions such as ionically crosslinked interactions, ${ }^{18}$ hydrogen bonding, ${ }^{19}$ and van der Waals interactions..$^{\mathbf{2 0 , 2 1}}$ The biomaterials such as agar, alginate, and cellulose can form strong physical interactions in hydrogels as a loosely linked network, and consequently, enhance their mechanical performance. Sun and co-workers ${ }^{22}$ reported about DN hydrogels composed of ionically crosslinked alginate and covalently crosslinked polyacrylamide, which could be stretched by $\sim 20$ times their original length and have fracture energies around $9000 \mathrm{Jm}^{2}$. Chen ${ }^{23}$ presents a one-pot method to prepare agar/polyacrylamide (agar/PAM) DN hydrogels. The tensile strength of agar/PAM DN hydrogels could reach ca. 1.0 MPa, and the maximum compression stress was $38 \mathrm{MPa}$. On the other hand, a tightly linked network is rigid and brittle, and it is formed by covalently crosslinked polyelectrolyte, which sustains the external force, maintain and recovery the shape of the hydrogel. ${ }^{24}$ Polyacrylamide hydrogels with $N, N^{\prime}$-methylenebisacrylamide as a crosslinking agent exhibit poor mechanical properties, limiting the mechanical properties of DN hydrogel to further improve. ${ }^{25}$ Therefore, design a tightly linked network of hydrogels may be a promising strategy for the enhancement of hydrogels' mechanical performance. ${ }^{26,27}$

Rosin is an abundant and low-cost natural product, and primarily consists of rosin acids with characteristic hydrophenanthrene structures and $\sim 10 \%$ neutral materials. ${ }^{28}$ Due to 
the hydrophenanthrene structures, rosin acids have cycloaliphatic and aromatic structures. ${ }^{29,30}$ It has been reported that polymers, such as epoxy resin, polyester and polyamide, containing rosin-based components have high thermal stability or great mechanical properties. ${ }^{31,32}$ For the cured acrylated epoxidized soybean oil, the rosin-based derivatives are significantly effect on improving the glass transition temperature and mechanical properties of the resin than the petroleum-based derivatives divinylbenzene. ${ }^{32}$ The rosin curing agent cured epoxies display the similar moduli and higher the glass transition temperature than the commercial monocyclic analogs. ${ }^{29,33}$ However, the rosin-based derivatives used in hydrogels to improve mechanical properties are rarely reported. In this study, the rosin-based monomer was synthesized and used as a crosslinking agent to fabricate DN polyacrylamide hydrogels. The RPAM hydrogels with the rosin-based crosslinking agent form both the chemical crosslinks and hydrophobic association crosslinked centers. The RPAM hydrogels were prepared via micellar copolymerization of acrylamide and rosin-based monomer in $7 \mathrm{wt} \%$ SDS aqueous solution. The swelling behavior, surface morphology, thermal stability, and mechanical properties of hydrogels were investigated. The polyacrylamide hydrogels with rosinbased monomer as crosslinking agent can withstand deformation such as ordinary stretching, knotted stretching, and compression, and exhibit high mechanical properties (elongation at break $\sim 1500 \%$, compressive strain $>95 \%$ ). The mechanical properties of RPAM hydrogels are significantly higher than hydrogels with $N, N^{\prime}$-methylenebisacrylamide as crosslinking agent reported in the literature. ${ }^{13,34,35}$ Therefore, these hydrogels as the tightly linked network show a great potential in the fabrication of hydrogels with excellent mechanical properties.

\section{Experimental section}

\subsection{Materials}

As previously described, ${ }^{36}$ fumaropimaric acid (FPA, GC purity 93\%) was obtained from rosin. Sodium dodecyl sulfate (SDS), acrylamide (AM, analytical grade), oxalyl chloride, acrylylchloride, ammonium persulfate (APS, analytical grade), and $N, N, N^{\prime}, N^{\prime}$-tetramethylethylenediamine (TEMED, analytical grade) were purchased from Aladdin Industrial Corporation (Shanghai, China); poly(ethylene glycol) 200 (PEG200, analytical grade) and tetrahydrofuran (analytical grade) were purchased from Energy Chemical (Shanghai, China).

\subsection{FPA-PEG200-AC preparation}

FPA was dissolved in tetrahydrofuran, and then, oxalyl chloride was added dropwise into the flask. The mixture was stirred at ambient temperature for $6 \mathrm{~h}$, and the excess oxalyl chloride and tetrahydrofuran were excluded under reduced pressure thereafter to obtain white solid FPA-Cl. FPA-Cl, triethylamine (TEA), and excess PEG 200 were mixed, and the solution was heated at $40{ }^{\circ} \mathrm{C}$ for $12 \mathrm{~h}$. Triethylamine hydrochloride was removed by filtration, and the organic phase was washed with dilute hydrochloric acid (three times), then with a $\mathrm{Na}_{2} \mathrm{CO}_{3}$ aqueous solution (three times), and subsequently dried with anhydrous sodium sulfate. Dichloromethane was evaporated under vacuum to yield a yellow viscous liquid, FPA-PEG200. FPA-PEG200, hydroquinone, TEA, and acryloyl chloride were added into a flask, and the resultant mixture was stirred at $40{ }^{\circ} \mathrm{C}$ for $12 \mathrm{~h}$. After the reaction, the organic phase was filtrated and washed with dilute hydrochloric acid (three times), then with a $\mathrm{Na}_{2} \mathrm{CO}_{3}$ aqueous solution (three times), and subsequently dried with anhydrous sodium sulfate. After evaporation of the solution, a yellow viscous liquid was obtained. The synthesis route of FPA-PEG200-AC is described in Scheme 1.

\subsection{RPAM hydrogel preparation}

RPAM hydrogels were prepared via free-radical micellar crosslinking copolymerization. $1.0 \mathrm{~g}$ AM was dissolved in $10.0 \mathrm{~mL}$ $7 \mathrm{wt} \%$ SDS aqueous solution. Various amounts of FPA-PEG200$\mathrm{AC}(1.5,2.0,2.5,3.0$, and $3.5 \mathrm{w} / \mathrm{v} \%)$ were dissolved in the resultant solution, and APS $(0.01 \mathrm{~g})$ and TEMED $(25 \mu \mathrm{L})$ were added into the solution afterward. The polymerization was conducted for $24 \mathrm{~h}$ at $35{ }^{\circ} \mathrm{C}$. To illustrate the synthetic procedure, we give details for the preparation of hydrogels with the FPA-PEG200-AC concentration of $2.5 \mathrm{w} / \mathrm{v} \%$ : first, $7 \mathrm{wt} \%$ concentration of SDS aqueous solution was prepared. And then, AM $(1.0 \mathrm{~g})$ and FPA-PEG200-AC $(0.25 \mathrm{~g})$ were dissolved in SDS aqueous solution $(10 \mathrm{~mL})$ under stirring and ultrasound to obtain a transparent solution. APS $(0.01 \mathrm{~g})$ and TEMED $(25 \mu \mathrm{L})$ were added into the solution afterward. Finally, the mixture was transferred into several sealable plastic tubes and the polymerization was conducted for $24 \mathrm{~h}$ at $35^{\circ} \mathrm{C}$.

\subsection{Solubilization of FPA-PEG200-AC in SDS solutions}

The solubilized amount of FPA-PEG200-AC in SDS micelles was calculated by measuring the transmittance of mixture solutions with a UV-visible spectrophotometer. The transmittance at a fixed wavelength was plotted as a function of the

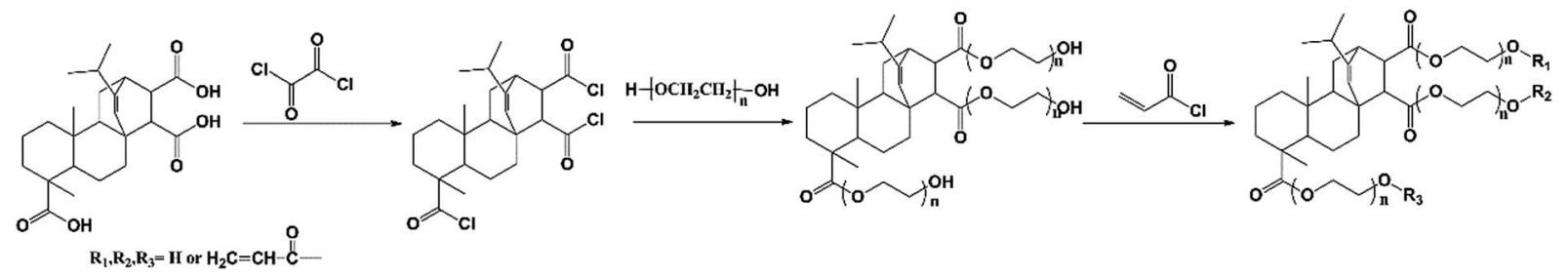

Scheme 1 Schematic for synthesis of FPA-PEG200-AC. 
solubilization amounts of FPA-PEG200-AC. The solubilization amount of FPA-PEG200-AC was determined by the curve break.

\subsection{Swelling measurements}

Swelling experiments were performed by immersing as-prepared cylindrical hydrogel samples (diameter $=6 \mathrm{~mm}$, length $=$ $10 \mathrm{~mm}$ ) in excess deionized water at $25^{\circ} \mathrm{C}$ until swelling equilibrium was attained. The swelling ratio (SR) was defined as follow:

$$
\mathrm{SR}=\frac{W_{\text {wet }}-W_{\text {dry }}}{W_{\text {dry }}}
$$

where $W_{\text {wet }}$ is the swollen weight of the hydrogel sample, while $W_{\text {dry }}$ is the dry weight of the hydrogel which the equilibrium swollen hydrogel lyophilized in freeze-drying equipment.

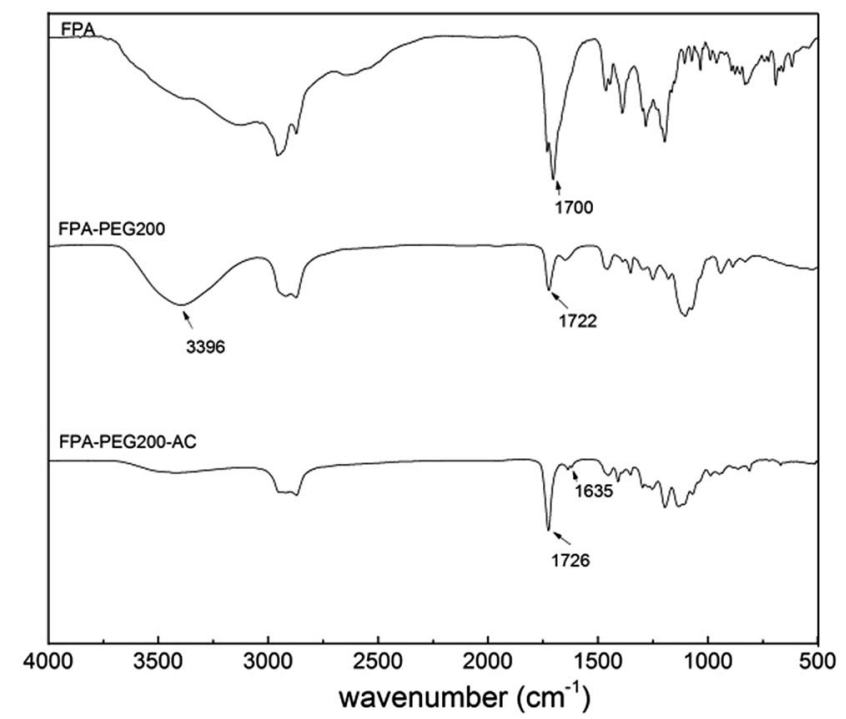

Fig. 1 The FT-IR spectra of FPA, FPA-PEG200, and FPA-PEG200-AC.

\subsection{Characterization}

${ }^{1} \mathrm{H}$ NMR spectra were recorded with a Bruker $300 \mathrm{MHz}$ spectrometer at room temperature with deuterated chloroform. FT-IR spectra were obtained using the Thermo Scientific Nicolet iS10 FT-IR spectrometer (Thermo Fisher Scientific, Waltham, MA, USA) in the wavenumber range of $400-4000 \mathrm{~cm}^{-1}$. Thermogravimetric analysis was performed using the STA409 synchronous thermal analyzer in a high-purity nitrogen atmosphere. Each sample was scanned from $30{ }^{\circ} \mathrm{C}$ to $800{ }^{\circ} \mathrm{C}$ at the heating rate of $10{ }^{\circ} \mathrm{C} \mathrm{min}^{-1}$. The surface morphology was observed using the 3400 NI scanning electron microscope. The freeze-dried hydrogels were coated with gold on the surface.

Tensile measurements were performed on cylindrical hydrogel samples $(6 \mathrm{~mm}$ diameter $\times 50 \mathrm{~mm}$ length) using the SANS electro-mechanical universal testing machine equipped with $250 \mathrm{~N}$ load cell. The measurement conditions are listed as follows: temperature of $25{ }^{\circ} \mathrm{C}$, gauge length of $30 \mathrm{~mm}$, and the crosshead speed of $10 \mathrm{~mm} \mathrm{~min}^{-1}$. The compressive measurements were performed on cylindrical hydrogels (35 mm diameter $\times 25 \mathrm{~mm}$ thickness) at the temperature of $25{ }^{\circ} \mathrm{C}$ and crosshead speed of $5 \mathrm{~mm} \mathrm{~min}^{-1}$.

\section{Results and discussion}

\subsection{Synthesis and characterization of FPA-PEG200-AC}

The FT-IR spectra of all the compounds occurring in the synthesis procedure are shown in Fig. 1. In FPA-PEG200 spectrum, the peaks of the ester carbonyl group at $1722 \mathrm{~cm}^{-1}$ and hydroxyl group at $3396 \mathrm{~cm}^{-1}$ suggest the esterification of FPA and PEG200. In FPA-PEG200-AC spectrum, size of the characteristic peak of hydroxyl group reduces, and the peak of terminal $\mathrm{C}=\mathrm{C}$ bonds at $1635 \mathrm{~cm}^{-1}$ appears. The ${ }^{1} \mathrm{H} \mathrm{NMR}$ spectrum of FPA-PEG200-AC is shown in Fig. 2. The peaks at 5.7-6.5 ppm are attributed to the protons on the unsaturated carbon of acrylic ester group. The peaks at 4.0-4.7 ppm and 3.5-

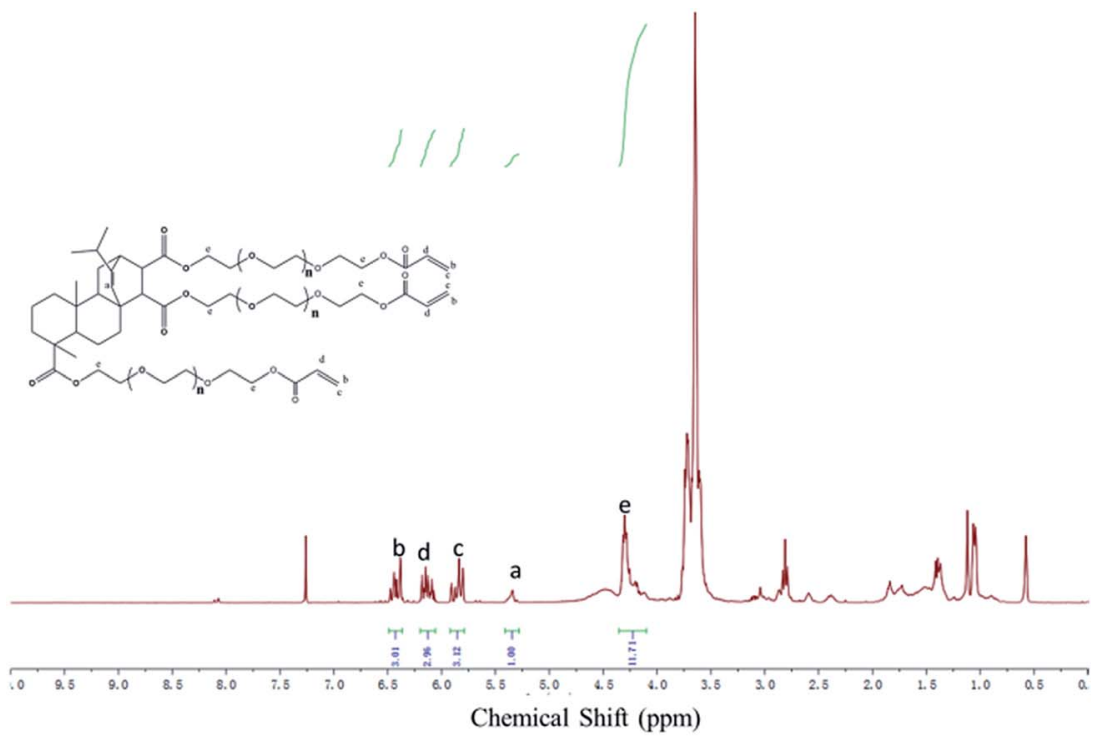

Fig. $2{ }^{1} \mathrm{H}$ NMR spectrum of FPA-PEG200-AC. 

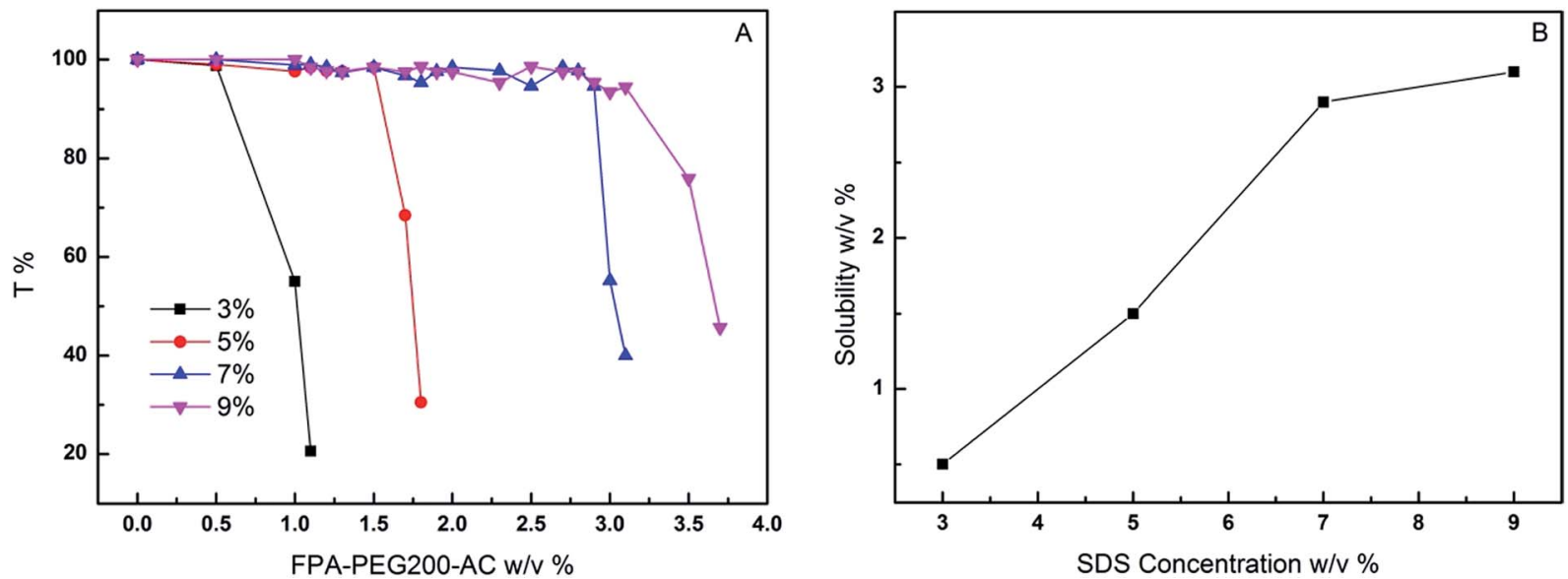

Fig. 3 (A) Plot of transmittance T vs. solubilization amount of FPA-PEG200-AC in different concentrations of SDS aqueous; (B) solubility of FPAPEG200-AC plotted against SDS concentration.

$4.0 \mathrm{ppm}$ are assigned to the protons on $-\mathrm{CH}_{2} \mathrm{O}-$ and $-\mathrm{COOCH}_{2}-$, respectively. The integrated area of signals was shown as follow:

$H_{\mathrm{a}}: H_{\mathrm{b}}: H_{\mathrm{c}}: H_{\mathrm{d}}: H_{\mathrm{e}}=1: 3.01: 3.12: 2.96: 11.71$.

The integrated values of the characteristic signals are well agreement with the theoretical values, which proved that a crosslinking agent molecular contains approximately three terminal acrylate groups.

\subsection{Solubilization of FPA-PEG200-AC in SDS micelles solution}

The concentrations of SDS aqueous were above the critical micellar concentration. Increasing number of the micelles also enhance the solubilization amount of FPA-PEG200-AC in aqueous. The transmittance curves of the solubilization
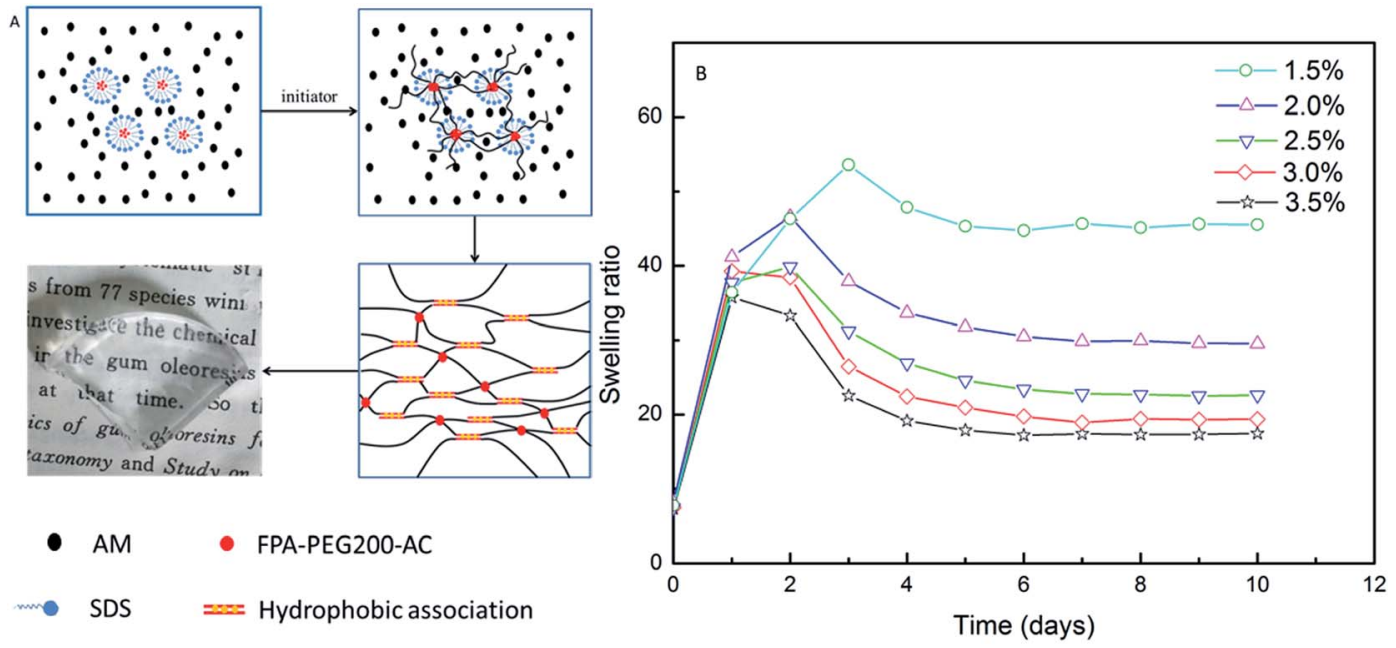

$1.5 \mathrm{w} / \mathrm{v} \% \quad 2.0 \mathrm{w} / \mathrm{v} \% \quad 2.5 \mathrm{w} / \mathrm{v} \% \quad 3.0 \mathrm{w} / \mathrm{v} \% 3.5 \mathrm{w} / \mathrm{v} \%$

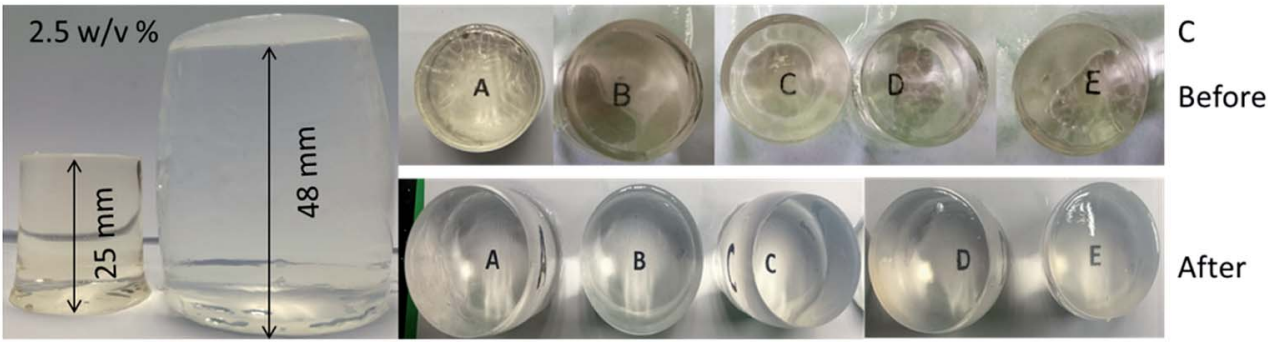

Fig. 4 (A) Schematic illustration of preparation and network of hydrogels (the transparent RPAM hydrogel (swollen state) with 1.5 w/v\% FPAPEG200-AC, $5 \mathrm{~mm}$ thickness); (B) swelling behavior of hydrogels with different concentrations of FPA-PEG200-AC; (C) optical images of the before and after swollen hydrogels. 

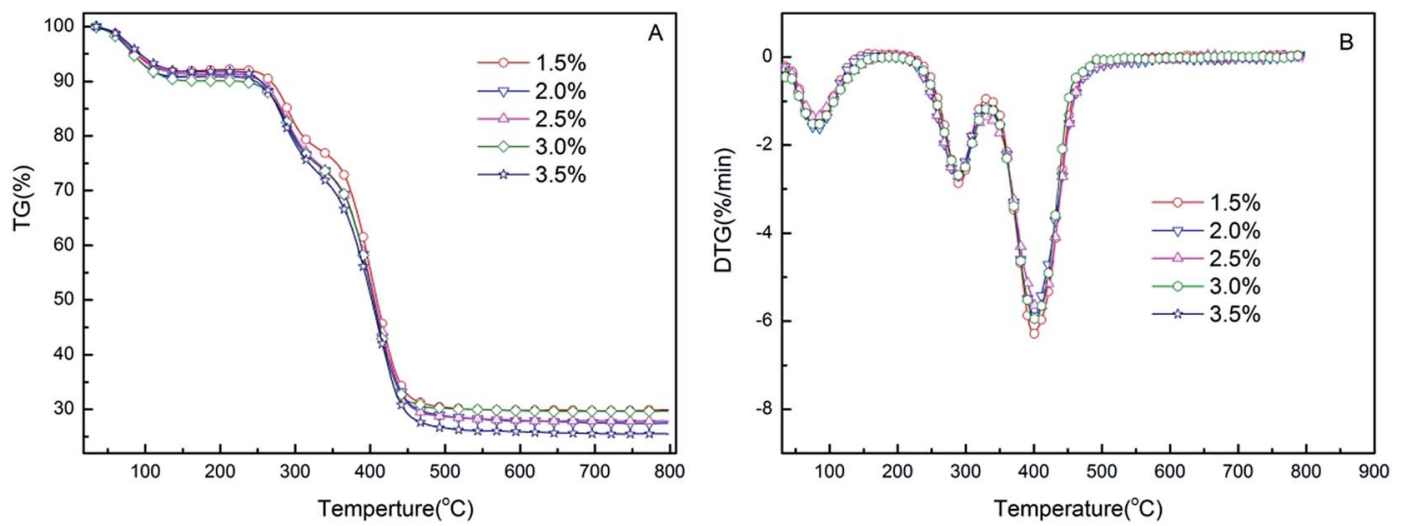

Fig. 5 Mass loss and thermal gravimetric curves of hydrogels with different FPA-PEG200-AC concentrations (w/v\%).

amounts of FPA-PEG200-AC as a function of concentrations of SDS aqueous are shown in Fig. 3A. The solubilization amounts of FPA-PEG200-AC are determined by the point on the graph where curve breaks. The solubility of FPA-PEG200-AC in different concentrations of SDS aqueous is presented in Fig. 3B. The solubilization amounts of FPA-PEG200-AC increase with an increase in the amount of SDS because of the rise in the number of micelles with an increase concentration of SDS. ${ }^{37}$ However, the solubilization amounts of FPA-PEG200-AC increase little when the concentration of SDS aqueous exceeds $7 \mathrm{wt} \%$. Thereby, we choose $7 \mathrm{wt} \%$ SDS aqueous for preparation of RPAM hydrogels.

\subsection{Hydrogel preparation and swelling behavior}

The RPAM hydrogels were prepared by micellar copolymerization of AM and FPA-PEG200-AC in SDS solution. The copolymerization mechanism and procedure to prepare the hydrogels are presented in Fig. 4A. In the micellar solution, the hydrophobic monomer FPA-PEG200-AC is solubilized in the SDS micelles and AM is dissolved in the aqueous phase. At first, $\mathrm{AM}$ is initiated by the initiator. The formed free radical encounters an SDS micelle, and as a result, the hydrophobic monomers are rapidly polymerized to form the crosslink structure of hydrogels. ${ }^{37}$

The swelling behaviors of the RPAM hydrogels at $25{ }^{\circ} \mathrm{C}$ are presented in Fig. 4B. The RPAM hydrogels swelled in water to reach a peak value of swelling ratio with increasing time. This is due to the osmotic pressure of SDS effect. In the initial swelling period, RPAM hydrogels contain a high concentration of SDS, resulting in a high osmotic pressure inside the gel network. ${ }^{15,38}$ Large amounts of water enter into the RPAM hydrogels system, which lead to a maximum value of swelling ratio. However, the swelling ratio of RPAM hydrogels decreased again until a swelling equilibrium was reached. The probable reason for this phenomenon is that as SDS is gradually extracted from the hydrogels, finally the osmotic effect disappear. ${ }^{38}$ Compared with
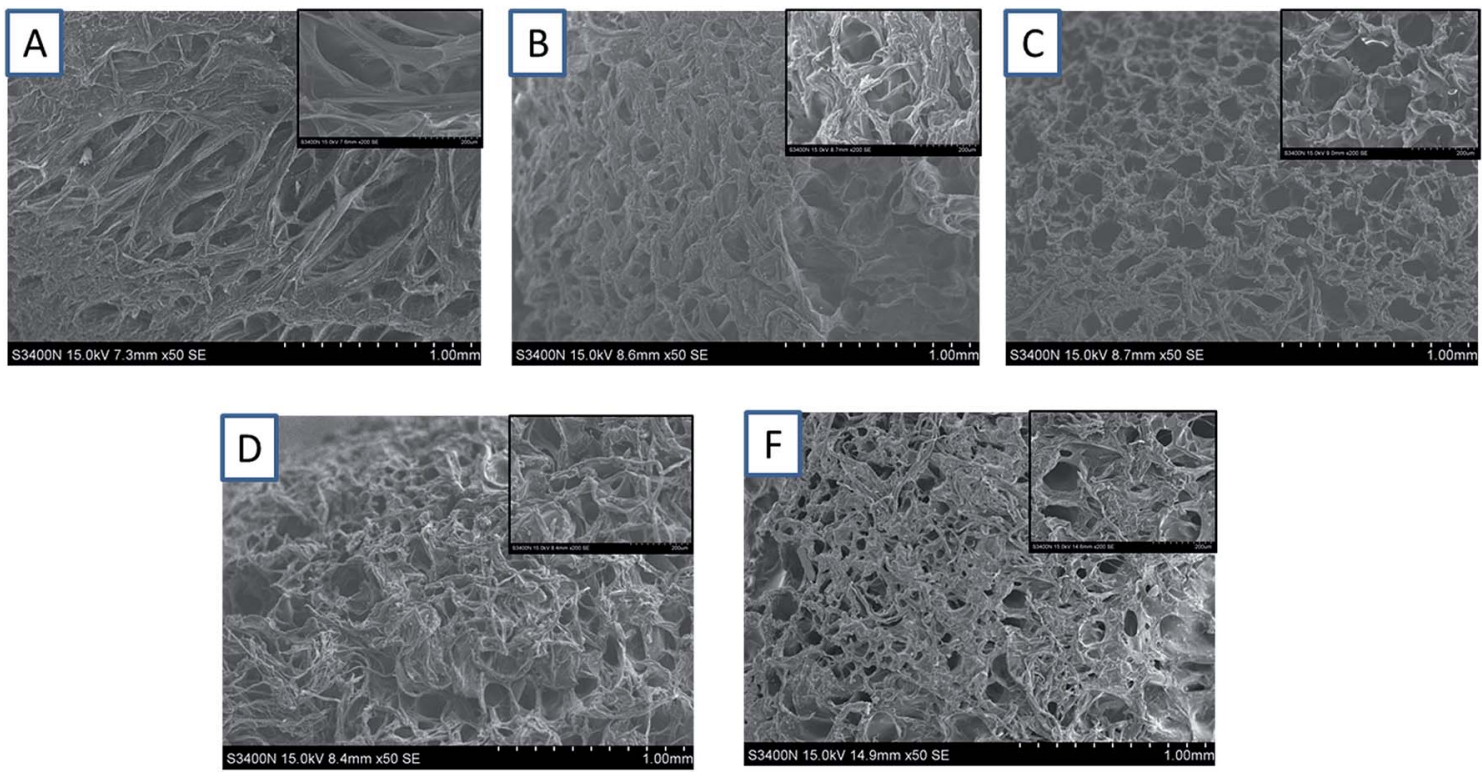

Fig. 6 SEM images of hydrogels with different FPA-PEG200-AC concentrations (w/v\%). (A) 1.5; (B) 2.0; (C) 2.5; (D) 3.0; and (E) 3.5 . 

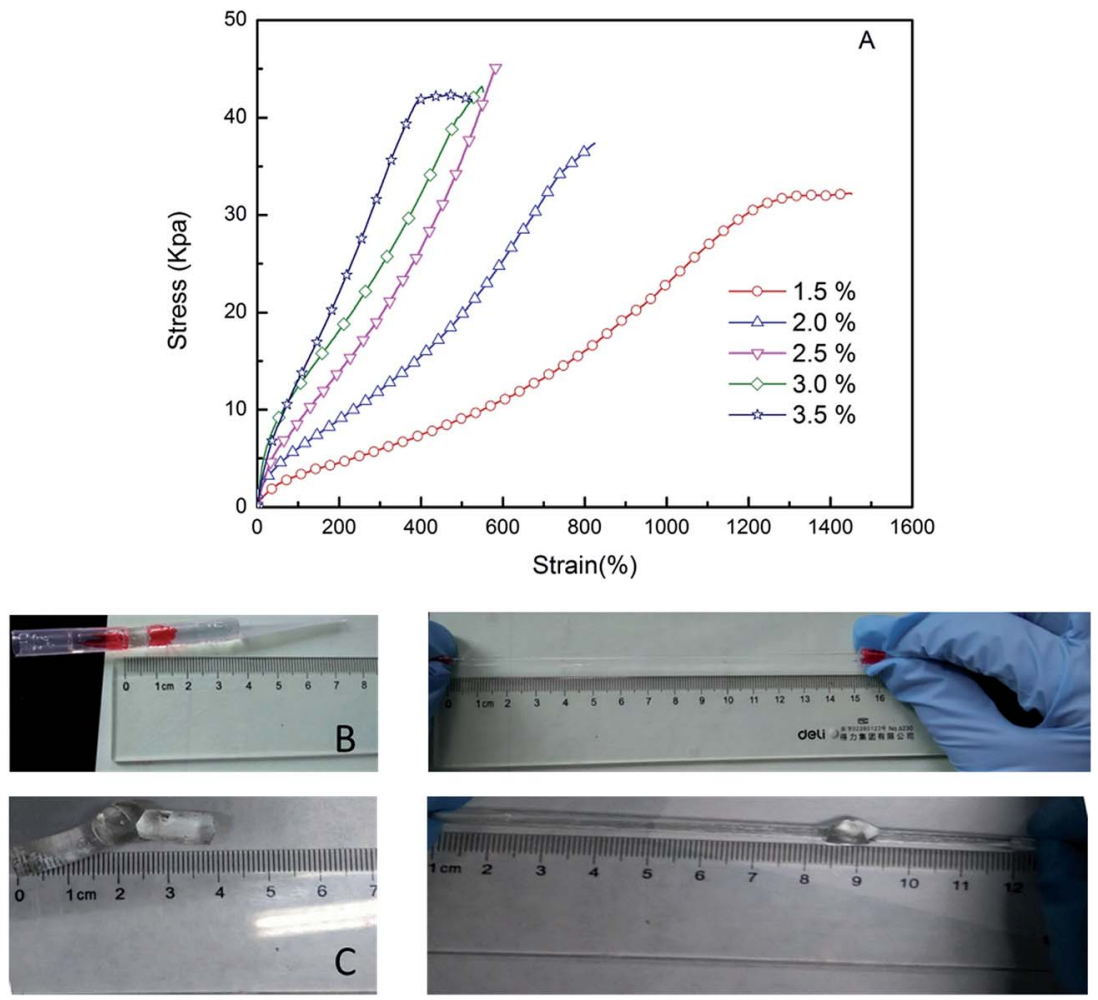

Fig. 7 (A) The stress-strain curves of hydrogels with various FPA-PEG200-AC concentrations (w/v\%); (B) ordinary stretching (RPAM hydrogels with $2.0 \mathrm{w} / \mathrm{v} \%$ FPA-PEG200-AC); (C) knotted stretching (RPAM hydrogels with $2.0 \mathrm{w} / \mathrm{v} \%$ FPA-PEG200-AC).

the hydrogels (HM-6) with $N$-hexyl-acrylamides as the hydrophobic comonomer and $N, N^{\prime}$-methylene-bis(acrylamide) as the crosslinking agent, ${ }^{15}$ the RPAM hydrogels exhibit a stronger swelling performance in the water. This may be owing to HM-6 with $N, N^{\prime}$-methylene-bis(acrylamide) as the crosslinking agent has a higher crosslinking density than RPAM hydrogels and the polyether structure in FPA-PEG200-AC weakened the hydrophobicity of hydrophenanthrene. However, compared with fully hydrophobically associated hydrogels (hydrogel (HM-C18) with stearyl methacrylate as the hydrophobic monomer; ${ }^{38}$ hydrogels (HA gels) with sodium 9 or 10-acrylamidostearic acid as the hydrophobic monomer ${ }^{34}$ ), the swelling performances of RPAM hydrogels are lower than HM-C18 and HA gels. This is due to the strength of physical crosslinking in HM-C18 and HA gels is weaker than the chemical crosslinking, resulting in a larger absorption of water. Besides, as the FPA-PEG200-AC concentration in hydrogels increases, the swelling ratio of hydrogels decreases. The result can be explained for two reasons. One is that the increases of crosslinking agent increase the crosslink density; another reason is the increase of hydrophobicity structure in the hydrogels lead to decreased water absorption. The optical images of the hydrogels are shown in Fig. 4C. The initial hydrogels with a light yellow is due to the yellow FPAPEG200-AC. The volume of swollen hydrogel with a high swelling ratio is much larger than the initial hydrogel. The initial and swollen hydrogels are transparent, but the swollen
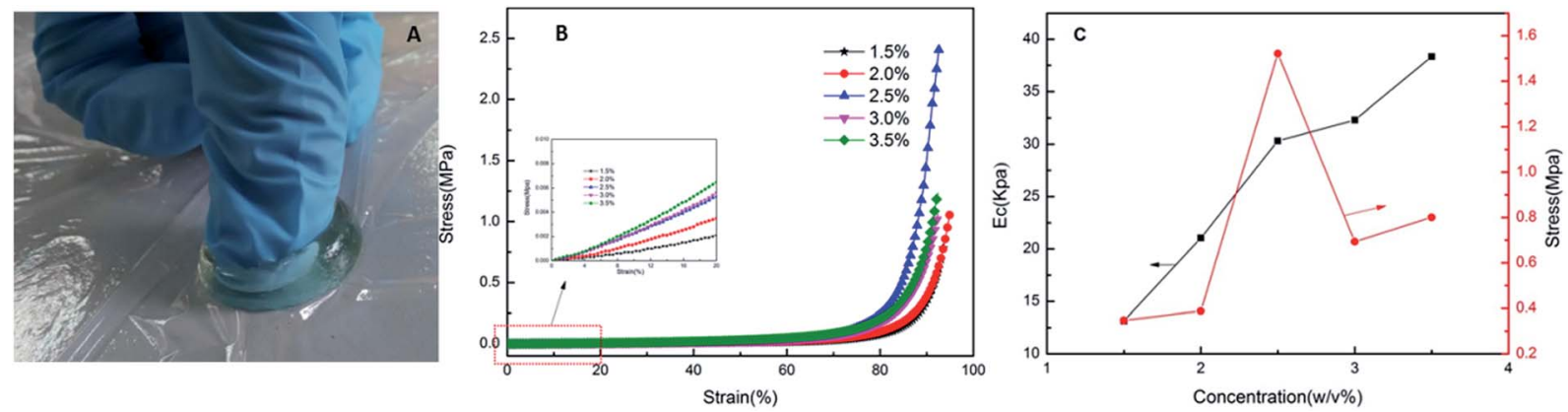

Fig. 8 (A) Finger compression; (B) compression stress-strain curves of hydrogels with various FPA-PEG200-AC concentrations (w/v\%); (C) elastic modulus of hydrogels and stress at $90 \%$ compression. 
hydrogel with $3.5 \mathrm{w} / \mathrm{v} \%$ FPA-PEG200-AC is blurred. It may be that the hydrogel system contains a large amount of hydrophobic monomers that exhibit microphase separation with the decrease of SDS.

\subsection{Thermal stability}

The mass loss and thermal gravimetric curves of RPAM hydrogels are shown in Fig. 5. The first step appears in the range of $25-150{ }^{\circ} \mathrm{C}$ with a mass loss of $6-9 \%$, which can be ascribed to the evaporation of intra- and inter-molecular moisture. The second step takes place in the range of $250-300{ }^{\circ} \mathrm{C}$ due to the decomposition of crosslinking components. The temperature of the RPAM hydrogels at the maximum rate of weight loss $\left(T_{\max }\right)$ reaches $290{ }^{\circ} \mathrm{C}$. The third step occurs in the range of $300-450{ }^{\circ} \mathrm{C}$ due to the degradation of primary chains. The hydrogels used rosin-based crosslinking agent show significantly higher thermal stability with the $T_{\max }$ of $400{ }^{\circ} \mathrm{C}$. This is because of the dominant effect of the thermally stable rosin structure and higher crosslink density. ${ }^{39}$

\subsection{SEM characterization}

The morphologies of RPAM hydrogels are shown in Fig. 6. The hydrogels' surface morphology shows a porous structure, which is because of the evaporation of water from the gels. The pore size of the gels decreases with the increasing concentration of FPA-PEG200-AC because the degree of crosslinking affects the porous size of the gels. As shown in Fig. 6C, at the FPA-PEG200AC concentration of $2.5 \mathrm{w} / \mathrm{v} \%$, the hydrogels have a regular porous size.

\subsection{Tensile properties}

The tensile properties of RPAM hydrogels are shown in Fig. 7. In general, the chemically crosslinked hydrogels exhibit poor mechanical properties because their structure lacks efficient energy dissipation mechanisms. The rosin-based crosslinking agent with the hydrophenanthrene structure has renowned hydrophobicity, and it can form hydrophobic association interaction. Thereby, the RPAM hydrogels form both the chemical crosslinks and hydrophobic association crosslinked centers. Under high-stress conditions, these hydrogels can sacrifice the hydrophobic association crosslinked centers to protect the chemically crosslinked units; hence, providing an effective way for energy dissipation. Consequently, the RPAM hydrogel structure is rebuilt after the stress is dissipated, and therefore, RPAM hydrogels have better mechanical properties. The hydrogels show high-level deformations of elongation and knotting (Fig. 7B and C). The RPAM hydrogels exhibit high tensile properties as the samples can be stretched by up to $1500 \%$ of their original lengths (see Fig. 7A). The shapes of the curves are nonlinear, and the elongation at break decreases with the increasing concentration of FPA-PEG200-AC, which is due to an increase in crosslink density.

\subsection{Compression properties}

Owing to efficient energy dissipation mechanisms, the RPAM hydrogels exhibit excellent compression properties. The RPAM hydrogels can withstand high strain without breaking (see Fig. 8A). In general, the PAM hydrogels with $N, N^{\prime}$-methylenebis(acrylamide) as a crosslinking agent fracture under the compressive deformation of $35-50 \% .{ }^{13,34,35}$ In this study, none of the RPAM hydrogels broke under high compressive strain (>95\%) and quickly recovered their original shape. When the strain exceeded $80 \%$, there was a rapid increase in stress (see Fig. 8B). The compressive elastic modulus (Ec), obtained from the slope at low deformation, increases with the increase in FPA-PEG200-AC concentrations (see Fig. 8C). At 90\% strain, stress for the hydrogels with the FPA-PEG200-AC concentration of $2.5 \mathrm{w} / \mathrm{v} \%$ reached a maximum value (Fig. $8 \mathrm{C}$ ). These results show that along with the degree of crosslinking, microstructure regularity also affects the compressive properties (see Fig. 5).

\section{Conclusions}

In summary, a novel type of hydrogels using a rosin-based crosslinking agent via micellar copolymerization were prepared, which can form both the chemical crosslinks and hydrophobic association crosslinked centers. Thereby, RPAM hydrogels exhibit high mechanical properties. The prepared hydrogels can withstand ordinary stretching, knotted stretching, and compression. The swelling ratio and elongation at break of these hydrogels decrease with the increase in the content of the crosslinking agent. At 90\% strain, the stress of RPAM hydrogels reaches maximum for the FPA-PEG200-AC concentration of $2.5 \mathrm{w} / \mathrm{v} \%$ because the pore size of these hydrogels reaches a regular value. This work can provide a new direction in the development of tough DN hydrogels via covalently crosslinked polyacrylamide networks.

\section{Conflicts of interest}

There are no conflicts to declare.

\section{Acknowledgements}

This study was supported by the National Natural Science Foundation of China (31470597).

\section{References}

1 X. W. Peng, L. X. Zhong, J. L. Ren and R. C. Sun, J. Agric. Food Chem., 2012, 60, 3909-3916.

2 L. Passauer, M. Struch, S. Schuldt, J. Appelt, Y. Schneider, D. Jaros and H. Rohm, ACS Appl. Mater. Interfaces, 2012, 4, 5852.

3 W. S. Wan Ngah, L. C. Teong and M. A. K. M. Hanafiah, Carbohydr. Polym., 2011, 83, 1446-1456.

4 S. Wan, S. Borland, S. M. Richardson, C. L. Merry, A. Saiani and J. E. Gough, Acta Biomater., 2015, 46, 29-40.

5 W. Shen, J. Luan, L. Cao, J. Sun, L. Yu and J. Ding, Biomacromolecules, 2015, 16, 105-115.

6 P. Calvert, Adv. Mater., 2009, 21, 743-756.

7 M. Z. Jing, Y. Fu, X. Fei, J. Tian, H. Zhi, H. Y. Zhang, L. Q. Xu, X. Y. Wang and Y. Wang, Polym. Chem., 2017, 8, 3553-3559. 
8 A. Dixit, D. S. Bag and S. J. S. Kalra, Polymer, 2017, 119, 263273.

9 D. C. Tuncaboylu, M. Sari, W. Oppermann and O. Okay, Macromolecules, 2011, 44, 4997-5005.

10 M. Tan, T. Zhao, H. Huang and M. Guo, Polym. Chem., 2013, 4, 5570 .

11 J. P. Gong, Y. Katsuyama, T. Kurokawa and Y. Osada, Adv. Mater., 2003, 15, 1155-1158.

12 K. Ito, Polym. J., 2007, 39, 489-499.

13 T. Huang, H. G. Xu, K. X. Jiao, L. P. Zhu, H. R. Brown and H. L. Wang, Adv. Mater., 2007, 19, 1622-1626.

14 K. Haraguchi and T. Takehisa, Adv. Mater., 2002, 14, 11201124.

15 S. Abdurrahmanoglu, V. Can and O. Okay, Polymer, 2009, 50, 5449-5455.

16 Q. Chen, L. Zhu, H. Chen, H. Yan, L. Huang, J. Yang and J. Zheng, Adv. Funct. Mater., 2015, 25, 1598-1607.

17 J. P. Gong, Soft Matter, 2010, 6, 2583-2590.

18 F. Luo, T. L. Sun, T. Nakajima, T. Kurokawa, Y. Zhao, A. B. Ihsan, H. L. Guo, X. F. Li and J. P. Gong, Macromolecules, 2014, 47, 6037-6046.

19 A. K. Gaharwar, S. A. Dammu, J. M. Canter, C. J. Wu and G. Schmidt, Biomacromolecules, 2011, 12, 1641.

20 T. Wang, S. Zheng, W. Sun, X. Liu, S. Fu and Z. Tong, Soft Matter, 2014, 10, 3506-3512.

21 H. Zhang, H. Peng, Y. Li, Y. Xu and W. Weng, Polymer, 2015, 80, 130-137.

22 J.-Y. Sun, X. Zhao, W. R. K. Illeperuma, O. Chaudhuri, K. H. Oh, D. J. Mooney, J. J. Vlassak and Z. Suo, Nature, 2012, 489, 133-136.

23 Q. Chen, L. Zhu, C. Zhao, Q. Wang and J. Zheng, Adv. Mater., 2013, 25, 4171-4176.
24 V. R. Tirumala, T. Tominaga, S. Lee, P. D. Butler, E. K. Lin, J. P. Gong and W. L. Wu, J. Phys. Chem. B, 2008, 112, 80248031.

25 J. Liang, G. Shan and P. Pan, Soft Matter, 2017, 13, 41484158.

26 T. Nakajima, Y. Fukuda, T. Kurokawa, T. Sakai, U.-I. Chung and J. P. Gong, ACS Macro Lett., 2013, 2, 518-521.

27 T. Sakai, T. Matsunaga, Y. Yamamoto, C. Ito, R. Yoshida, S. Suzuki, N. Sasaki, M. Shibayama and U. I. Chung, Macromolecules, 2008, 41, 5379-5384.

28 B. K. Yadav, B. Gidwani and A. Vyas, J. Bioact. Compat. Polym., 2015, 31, 111-126.

29 X. Liu, W. Xin and J. Zhang, Green Chem., 2009, 11, 1018.

30 P. A. Wilbon, F. Chu and C. Tang, Macromol. Rapid Commun., 2013, 34, 8-37.

31 H. Zhang, Y. Yang, H. Liu, J. Song, S. Shang and Z. Song, Front. Arch. Res., 2017, 4, 97-105.

32 Q. Ma, X. Liu, R. Zhang, J. Zhu and Y. Jiang, Green Chem., 2013, 15, 1300.

33 H. Wang, X. Liu, B. Liu, J. Zhang and M. Xian, Polym. Int., 2009, 58, 1435-1441.

34 W. Li, H. An, Y. Tan, C. Lu, C. Liu, P. Li, K. Xu and P. Wang, Soft Matter, 2012, 8, 5078.

35 J. Fan, Z. Shi, M. Lian, H. Li and J. Yin, J. Mater. Chem. A, 2013, 1, 7433.

36 X. Xu, Z. Song, S. Shang and X. Wang, Chem. Ind. For. Prod., 2009, 29, 69-72.

37 B. Gao, H. Guo, J. Wang and Y. Zhang, Macromolecules, 2008, 41, 2890-2897.

38 D. C. Tuncaboylu, M. Sahin, A. Argun, W. Oppermann and O. Okay, Macromolecules, 2012, 45, 1991-2000.

39 J. Yu, Y. Liu, X. Liu, C. Wang, J. Wang, F. Chu and C. Tang, Green Chem., 2014, 16, 1854. 